JOURNAL DE PHYSIQUE IV

Colloque C7, supplément au Journal de Physique III, Volume 4, juillet 1994

\title{
Purely thermal wave based non-chemical photopyroelectric gas sensor: application to hydrogen
}

\author{
M. Munidasa, A. Mandelis and A. Katz \\ Photothermal and Optoelectronic Diagnostic Laboratory, Department of Mechanical Engineering and \\ Center for Hydrogen and Electrochemical Studies (CHES), University of Toronto, Toronto, Canada, \\ MSS $1 A 4$
}

\begin{abstract}
A commercially available polyvinylidene fluoride (PVDF) pyroelectric film with an optically generated thermal wave field has been introduced as the active device for a new nonchemical hydrogen gas sensor. The sensitivity to hydrogen at low flow rates $(<500 \mathrm{ml} / \mathrm{min}$.) is obtained through thermal boundary condition changes introduced by the gas at the film-gas interface. Photopyroelectric voltage amplitude and phase changes due to hydrogen-air mixtures with respect to pure air, obtained through a lock-in amplifier are discussed.
\end{abstract}

\section{INTRODUCTION}

In recent years, hydrogen has grown to be one of the most useful gases, leading to considerable research efforts towards the development of hydrogen gas sensors. Most of the detection methods are based on electronic devices and use palladium as the active surface element. These devices make use of one or more of the many different effects caused by the absorption and desorption of hydrogen in Pd. Most of these sensors were semiconductor-based[1-4] and require fabrication based on sophisticated, multiple-step processing. They only behave essentially reversibly under specific conditions of pressure, temperature, geometry, etc. During the last few years, much progress has been made in this laboratory in developing a new photopyroelectric hydrogen gas sensor[5] using Pd-coated pyroelectric thin films, which has distinct advantages over other sensors under STP conditions[6,7]. The hypothesis was that, upon establishing an ac steady-state temperature (thermal-wave) field within the pyroelectric electret by intensity-modulated laser irradiation, followed by absorption and photothermal energy conversion, any changes in the pyroelectric generation mechanism(s) in the polyvinylidene fluoride film (PVDF), due to interactions with the Pd-absorbed hydrogen, would be registered as changes in the observed pyroelectric signal due to a thermodynamically established potential difference between active ( $\mathrm{Pd}$ ) front and passive ( $\mathrm{Ni}-\mathrm{Al}$ ) back electrodes, thus yielding a hydrogen sensor. In this paper we describe a novel non-chemical evolution of this detector, where any inexpensive metal electrode (such as aluminum) can be used instead of Pd. This new sensor is based purely on the difference in thermophysical properties between the detected gas and the ambient. The difference in thermal conductivity of gases has been utilized for decades in gas chromatography(GC) in the form of a thermal conductivity detector(TCD)[8]. In the TCD, the change in resistivity due to change in temperature of the hot filament is measured using a Wheatstone bridge. A carrier gas (usually helium or hydrogen) with high thermal conductivity is passed through a cell where the filament 
is placed. When a small amount of second gas of lower thermal conductivity is introduced into the flow, the temperature of the filament increases due to the poorer heat loss into the gas. Since this is purely a dc measurement, the signal is effected by drift, temperature fluctuations of the cell, environment and sensing elements, by turbulence of the gas flow and by mechanical vibrations[9]. Traces of oxygen in the carrier gas should be carefully eliminated in order to avoid rapid oxidation of the filament[9]. Therefore, this device is not suitable for reliable, reversible, long-term continuous gas detection in an open environment. In a typical TCD, with He carrier gas and a filament current of $175 \mathrm{~mA}$, the filament may reach an equilibrium temperature of $100^{\circ} \mathrm{C}$ above the surrounding block temperature. This feature may make it unsafe for hydrogen detection, especially near the critical hydrogen-oxygen mixture, where possible ignition and explosion is of great concern for practical applications.

\section{EXPERIMENTAL AND DISCUSSION}

The basic cross-sectional geometry of the sensor is shown in Fig.1. A commercial PVDF film[10], $9 \mu \mathrm{m}$ thick with $\mathrm{Ni} / \mathrm{Al}$ electrodes on both sides was installed in a commercially available INFICON ${ }^{\mathrm{TM}}$ housing (Fig.1(a)). Light from an intensity-modulated laser diode with variable frequency was guided on to the back of the film using an optical fiber. The sensor was placed inside a chamber which permitted the controlled, continuous flow of gas over the sensor. The signal from the film was preamplified and then connected to a lock-in amplifier, interfaced to a computer to record both amplitude and phase.

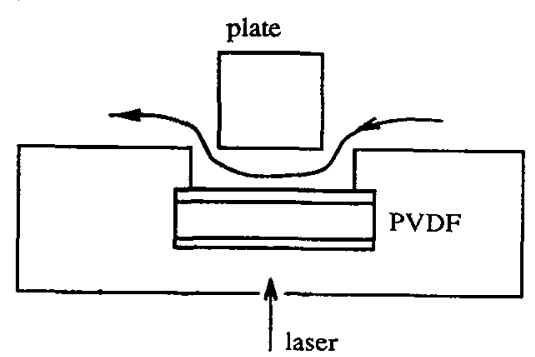

Cross-sectional view

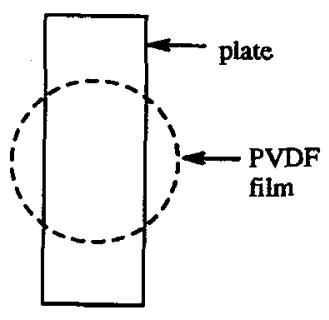

Top view

(a)

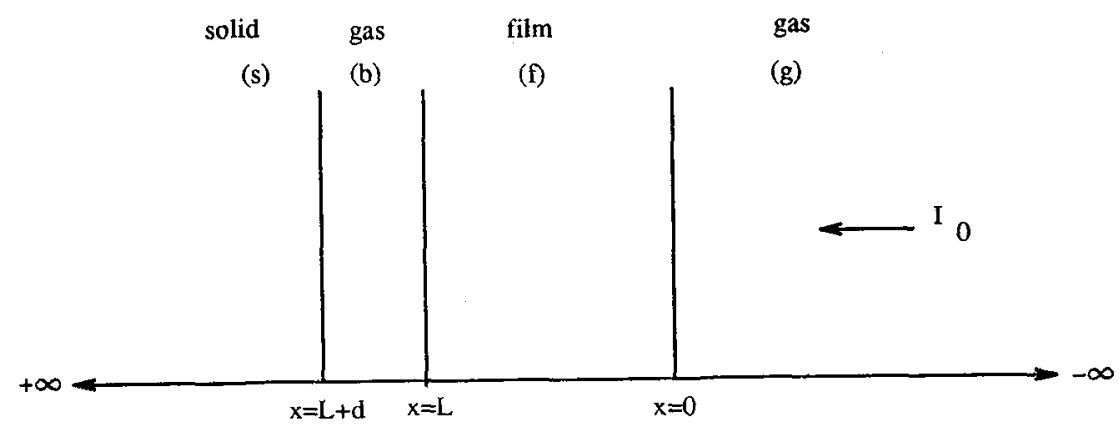

(b)

Figure 1. (a) Cross-sectional view and top view of the PVDF film with a plate (cap) over it. (b) Basic geometry of the sensor. PVDF film (f) of thickness $L$ with a semi-infinite region of gas on the laser side (g) and a gas layer (b) of thickness $\mathrm{d}$ on the opposite side, followed by a semi-infinite region of solid (s). 
The pyroelectric voltage which is proportional to the average temperature of the film for a given gas can be calculated by solving coupled one-dimensional heat diffusion equations subject to a harmonically oscillating thermal flux $\mathrm{F}_{0}(\omega)=\eta \mathrm{I}_{0}[1+\exp (\mathrm{i} \omega \mathrm{t})] / 2$ at the PVDF-gas interface $\mathrm{x}=0$, with appropriate boundary conditions, in each region (g), (f), (b) and (s) (Fig.1(b)). Here $\eta \simeq 1$ is the optical-to-thermal energy conversion efficiency of the PVDF surface electrode exposed to the laser beam, $I_{0}$ is the optical irradiance and $\omega$ is the beam intensity modulation angular frequency. Although our experiments were performed under a continuous flow of gas $(<510 \mathrm{ml} / \mathrm{min})$, here we assume that the gas is motionless. In fact, experiments have clearly shown that the flow rate of hydrogen does not affect the saturation magnitude of the signal. It only changes the rate at which the signal reaches its maximum. It has been found that by restricting the gas flow over the PVDF film opposite to the laser side to a thin layer smaller than the thermal diffusion length $\mu$ in the sensing gas, sensitivity of the phase of the signal to the type of ambient gas is greatly enhanced. The thermal diffusion length $\mu$ is given by $\mu=(2 \alpha / \omega)^{1 / 2}$ where $\omega$ is the angular modulation frequency of the laser intensity and $\alpha$ is the thermal diffusivity of the gas. This geometry is achieved by placing a solid plate over the detector as shown in Fig.1(a). In our setup the gap between the plate and the surface of the film is $0.5 \mathrm{~mm}$ (Thermal diffusion length in hydrogen at $11 \mathrm{~Hz}$ is $2.3 \mathrm{~mm}$ ). The sensitivity of the detector increases with decreasing film thickness in both amplitude and phase channels. With a $28 \mu \mathrm{m}$-thick PVDF film we were able to detect only down to $5 \%$ hydrogen in air, and $1 \%$ with a $9 \mu \mathrm{m}$-thick film. At these low concentrations signal changes due to hydrogen were observed only in the phase channel.

Figure 2 shows data taken immediately after a new $28 \mu \mathrm{m}$-thick film was inserted in the housing. Several cycles of $100 \%$ hydrogen and $100 \%$ air are shown with a capped PVDF film at $21 \mathrm{~Hz}$. These data show that the film responds immediately to hydrogen whereas in the Pd-PVDF chemical sensor several cycles of hydrogen exposure are required until a stabilized response is achieved. Furthermore, the phase of the signal shows excellent quality, reproducibility and reversibility, but the amplitude drifts. Data taken several hours later showed that the amplitude has stabilized. Here as well the phase data was far superior and has not drifted or changed in magnitude during the idle period.

Figure 3 (solid line) shows the phase of the PPE signal for $100 \%$ helium relative to pure air. The corresponding data for $100 \%$ hydrogen are shown as a dotted line for comparison. The change in phase due to helium is $\mathrm{ca} .81 \%$ of the phase change due to hydrogen; theory predicts a change of $79 \%$ $\left(\mathrm{k}_{\mathrm{He}}=152 \times 10^{-3} \mathrm{~W} / \mathrm{mK}, \alpha_{\mathrm{He}}=180 \times 10^{-6} \mathrm{~m}^{2} / \mathrm{s}, \mathrm{k}_{\mathrm{H}}=182 \times 10^{-3} \mathrm{~W} / \mathrm{mK}\right.$ and $\alpha_{\mathrm{H}}=155 \times 10^{-6} \mathrm{~m}^{2} / \mathrm{s}$ from Ref.11). The discrepancy may be due to the three-dimensionality of the heat flow in the experiment where the optical fiber was very close to the film.

\section{CONCLUSION}

The novel hydrogen sensor described in this paper has shown reliability, reversibility and excellent stability against drift in the thermal-wave phase detection mode. No hot igneous filament or other combustible elements are involved and, therefore, the new sensor is a good candidate for safe hydrogen detection in open ambients. The PVDF device is based on ac thermal-wave propagation in the bulk of the pyroelectric element and the signal is measured at a fixed frequency, thus eliminating ambient transient thermal effects and dc drifts. It is also immune to background temperature fluctuations or mechanical vibrations. The temperature elevation of the PVDF sensor film due to laser heating is extremely small $(<1 \mathrm{~K})$, a feature which gives the new sensor a definite advantage for safe hydrogen sensing. Irreversible sensitivity loss with each successive introduction of hydrogen up to several exposures reported for Pd-based chemical sensors[12] is not present in this detector. Therefore, it is not necessary to reactivate the sensor after a prolonged idle time. Since any inexpensive metal can be used as electrodes, the cost of the sensor is greatly reduced. A sheet of 9 $\mu \mathrm{m}$ thick $15 \mathrm{~cm} \times 15 \mathrm{~cm}$ PVDF with $\mathrm{Ni} / \mathrm{Al}$ electrodes which can supply 200 sensor elements costs $\$ 55$ (1992-93). We have demonstrated two ways of improving the sensitivity of the sensor, (1) by placing a cap over the film so that the gas flow over the detector is restricted to a thin layer, and (2) by using 
a thinner film. The data strongly suggest that the phase of the signal should be the preferred channel in terms of stability. Although the sensitivity of this sensor is not as good as the Pd-based sensor, a reasonably good sensitivity of $1 \%$ hydrogen in air has been achieved. It is important to note that this concentration (1\%) is not believed to be an absolute minimum. A very recent optimization of our setup exhibited improved performance with sensitivity better than $0.5 \%$.

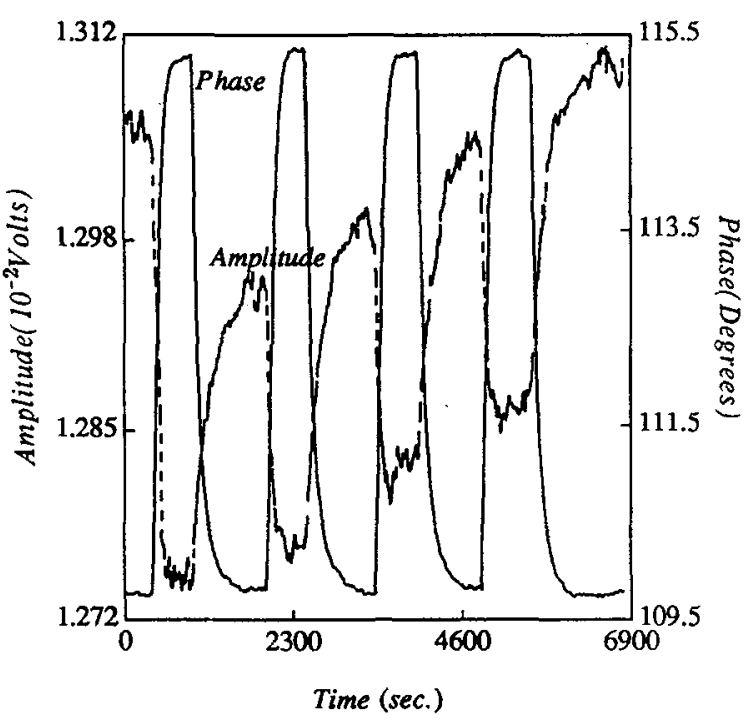

Figure 2. The amplitude (dashed) and the phase (solid) of the PPE signal for the first four cycles of pure hydrogen and pure air immediately after inserting a fresh film of thickness $28 \mu \mathrm{m}$.

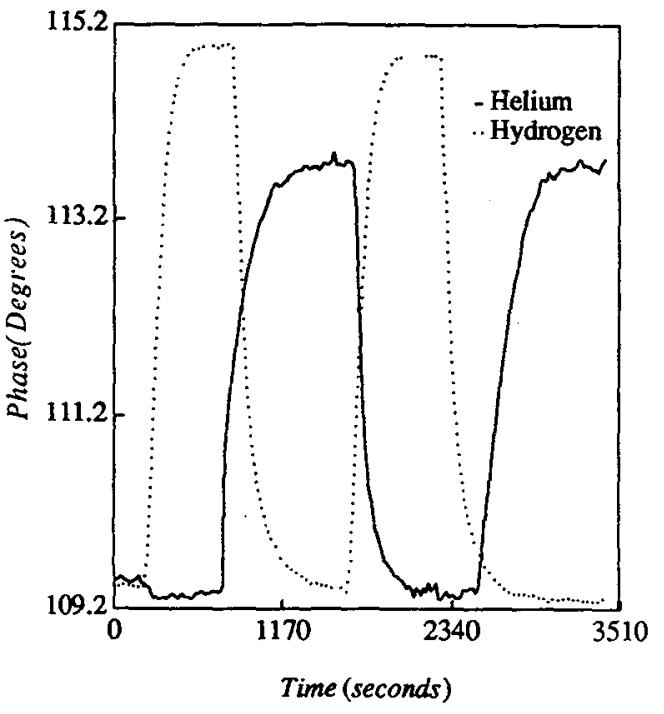

Figure 3. The phase of the signal for pure helium (solid line) compared to pure hydrogen (dotted line) and air. Both baselines correspond to $100 \%$ ambient air.

\section{REFERENCES}

1. K.I. Lundstrom, M.S. Shivaraman and C.M. Svensson, Surf.Sci., 64, (1977) 497.

2. M.C. Steele and B.A. Maclver, Appl.Phys.Lett., 28, (1976) 687.

3. A.D'Amico, G. Fortunato and G. Petrocco, Sensors and Actuators, 4, (1983) 349.

4. T.L. Poteat and B. Lalevic, IEEE Trans.Electron Dev. ED-29, (1982) 123.

5. A. Mandelis and C. Christofides, Sensors and Actuators B2, (1990) 79.

6. C. Christofides and A. Mandelis, J.Appl.Phys., 68, (1990) R1.

7. A. Mandelis and C. Christofides, Physics, Chemistry and Technology of Solid State Gas Sensor Devices, (J.D. Winefordner, Ed., J. Wiley, New York, 1993) 253-308.

8. G.A. Shakespear, Proc. Phys. Soc. London, 33, (1921) 163.

9. G. Guiochon and C. L. Guillemin, Quantitative Gas Chromatography for laboratory analyses and on-line process control, J. Chrom. Lib. 42, (Elsevier, New York, 1988) 423.

10. KYNAR Piezo Film Technical Manual, Pennwalt Corp., King of Prusia, PA (1983).

11. J.H. Lienhard, A Heat Transfer Textbook, (Prentice-Hall Inc., New Jersey 1981) 494-501.

12. C. Christofides and A. Mandelis, J.Appl.Phys. 66, (1989) 3975. 